\title{
Measurement of jet production properties with the ATLAS detector
}

\author{
Camille BELANGER-CHAMPAGNE* \\ on behalf of the ATLAS Collaboration \\ $M c$ Gill University (CA) \\ E-mail: camille@physics.mcgill.ca
}

Measurements of the production cross sections of inclusive, di- and tri-jet events probe the dynamics of QCD and can constrain the parton proton structure. Double-differential cross sections are measured with the ATLAS detector at the LHC and compared to expectations based on nextto-leading order QCD calculations. These measurements constitute precision tests of QCD in the new energy regime available at the LHC from proton-proton collision centre-of-mass energy of $7 \mathrm{TeV}$. The study of di-jet systems is sensitive to physics beyond the Standard Model in the form of an effective model of contact interactions. Limits on the compositeness scale parameter are measured to be in the 6.9 to $7.7 \mathrm{TeV}$ range.

XXIII International Workshop on Deep-Inelastic Scattering

27 April - May 12015

Dallas, Texas

${ }^{*}$ Speaker. 


\section{Introduction}

The ATLAS collaboration has now performed precision measurements of jet production crosssections for inclusive jet production as well as di- and tri-jet events using all the data it collected in 2011 from proton-proton collisions with a centre-of-mass energy of $7 \mathrm{TeV}$ at the Large Hadron Collider. This data sample far exceeds the statistical power of the previous 2010 data sample. Furthermore, the precision of these new results benefits from reduced systematic uncertainty contributions. Double-differential cross-sections are measured for many jet final state configurations over a broad partonic centre-of-mass energy range and compared to next-to-leading order (NLO) quantum chromodynamics (QCD) calculations. A search for new physics in the framework of contact interactions is performed using the di-jet double-differential cross-section measurement.

\section{Data samples and theoretical predictions}

The data used to obtain the cross-section measurements presented here was collected during 2011 with the ATLAS detector [1] and the dataset has a total luminosity of $4.5 \mathrm{fb}^{-1}$. The analyses use data collected from a combination of jet-based trigger requirements where each trigger requirement is measured to be fully efficient over the jet transverse momentum $\left(p_{\mathrm{T}}\right)$ range where it is used for data acquisition. Jets are reconstructed from energy clusters in the ATLAS calorimeter using the anti- $\mathrm{k}_{t}$ algorithm [2] with size parameter values of $\mathrm{R}=0.4$ and $\mathrm{R}=0.6$ in the rapidity region $|y|<3$.

Theoretical predictions at parton-level are calculated at fixed-order in perturbative QCD (pQCD) using NLOJET++ [3]. Results are interfaced with APPLGRID [4] for fast convolution with multiple sets of parton distribution functions (PDFs) including CT10 [5], HERAPDF1.5 [6], MSTW2008 [7], NNPDF2.1/2.3 [8] and ABM11 [9]. An electroweak correction based on [10] is applied and non-perturbative corrections for fragmentation and hadronization effects are calculated using Pythia [11] and Herwig [12], resulting in a particle-level theoretical prediction.

To perform comparisons between data measurements and theoretical predictions, data distributions are unfolded to particle-level by correcting for detector inefficiencies and resolution effects using the Iterative, Dynamically Stabilised Bayesian unfolding method [13].

\section{Inclusive jet cross-section}

The inclusive jet cross-section [14] is measured as a function of jet $p_{\mathrm{T}}$ for jets with $p_{\mathrm{T}}>$ $100 \mathrm{GeV}$ in rapidity regions $|y|$ of size 0.5 . Results are shown in Figure 1 for jets with size parameter $\mathrm{R}=0.4$ and $\mathrm{R}=0.6$. The results are compared to NLO $\mathrm{pQCD}$ predictions obtained using CT10. The experimental systematic uncertainties are dominated by the uncertainty on the jet energy scale correction. The uncertainty on the $p_{\mathrm{T}}$ of a jet is below $2 \%$ in the central detector region but increases to $4 \%$ in the forward region for jets with $p_{\mathrm{T}}$ around $100 \mathrm{GeV}$. Other significant sources of uncertainty include the jet energy resolution and angular resolution, the jet reconstruction efficiency, the unfolding procedure and the measurement of the luminosity of the data sample. The uncertainty on the luminosity is $1.8 \%$ for this analysis as well as all other analyses presented here.

The NLO pQCD predictions reproduce well the behaviour of the measured cross-sections over eight orders of magnitude in the measured cross-section and in all six rapidity bins. A subset of 


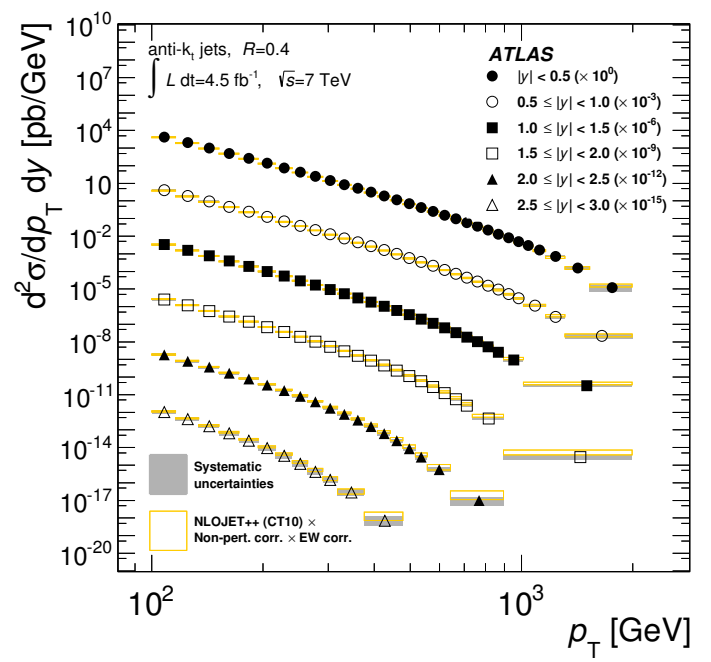

(a)

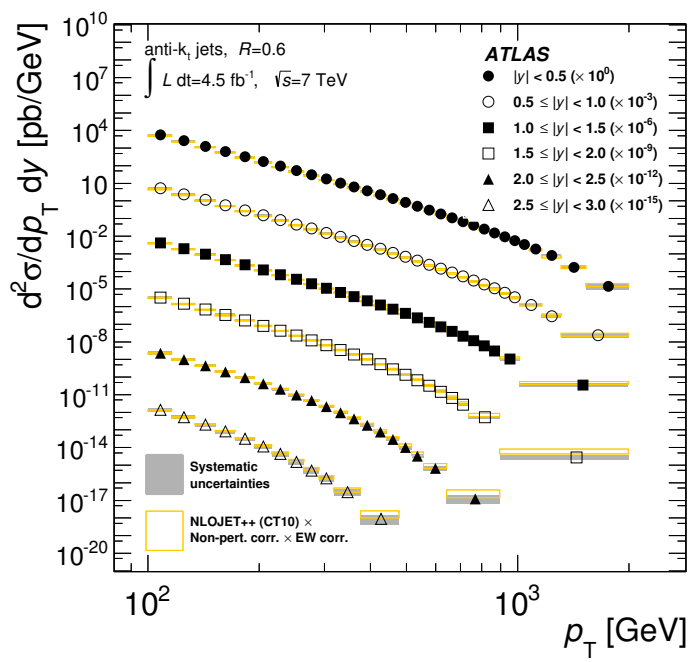

(b)

Figure 1: Double-differential inclusive jet cross-section as a function of jet $p_{\mathrm{T}}$ in bins of absolute rapidity $|y|$ for jets of size parameter (a) $\mathrm{R}=0.4$ and (b) $\mathrm{R}=0.6$. Each rapidity bin is multiplied by a power of 10 for legibility. Statistical uncertainties are smaller than the data points while systematic uncertainties are indicated by a grey box. NLOJET++ pQCD predictions with their uncertainties are shown as yellow open boxes [14].
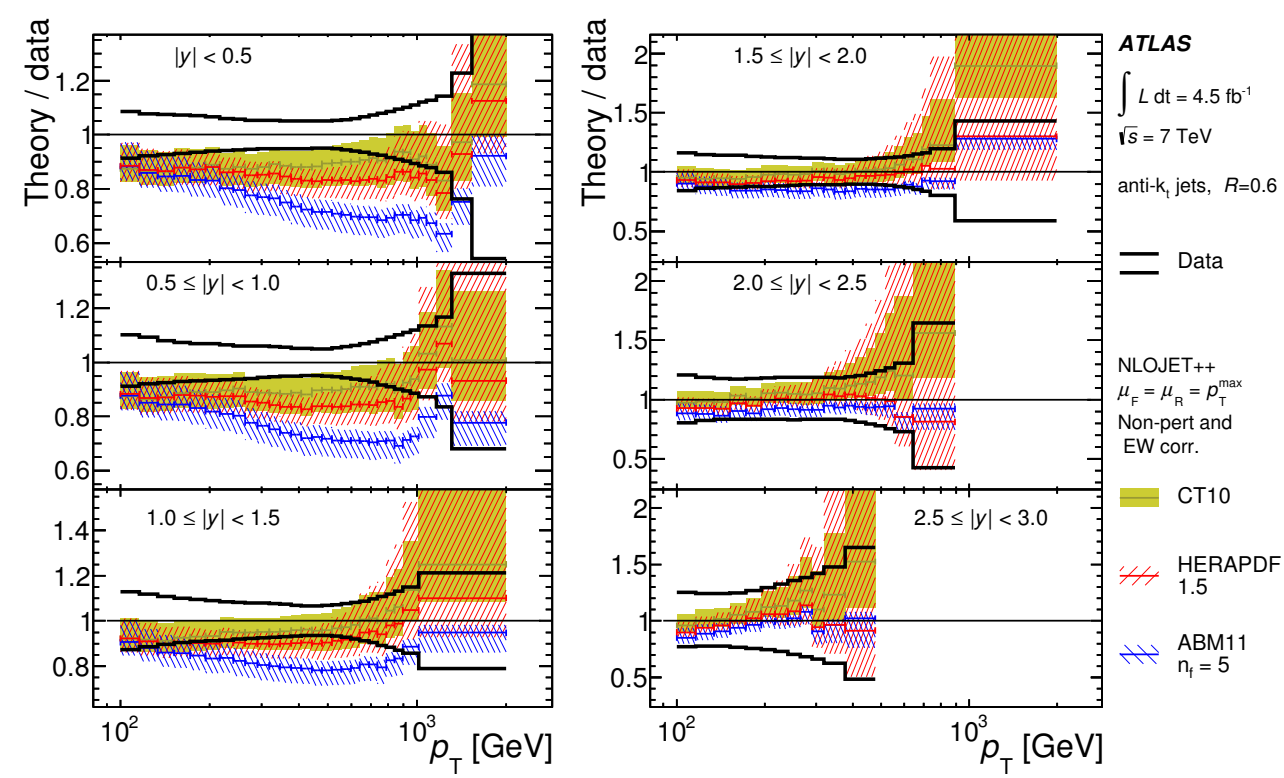

Figure 2: Ratio of NLO pQCD predictions, obtained with different PDF sets, to the measured doubledifferential inclusive jet cross-section, shown as a function of the jet $p_{\mathrm{T}}$ in six rapidity bins for jets with size parameters $\mathrm{R}=0.6$. The PDF sets used are CT10 (yellow), HERAPDF 1.5 (red) and ABM11 (blue). For both measurements and predictions, the sum of all uncertainties considered is shown as a band except for the uncertainty on the luminosity of the dataset which is not included in the black lined band [14]. 
the comparisons done with NLO pQCD predictions obtained using other PDF sets is shown in Figure 2 for jets with $\mathrm{R}=0.6$. For both jet sizes, the predictions are generally consistent with the measured cross-sections. For jets with size parameter $\mathrm{R}=0.6$, the predictions tend to be systematically lower than the measurements in the lowest rapidity regions. Predictions using ABM11 are in disagreement with data in the three lowest rapidity regions for both jet sizes.

\section{Di-jet cross-section}

The double differential di-jet cross-section [15] as a function of the mass of the two leading $p_{\mathrm{T}}$ jets $m_{12}=\sqrt{\left(p_{1}^{2}+p_{2}^{2}\right)}$ is measured in six slices of the half rapidity separation $y^{*}=\left|y_{1}-y_{2}\right|$. The $p_{\mathrm{T}}$ of the leading jet must be $>100 \mathrm{GeV}$ while the subleading jet must have $p_{\mathrm{T}}>50 \mathrm{GeV}$. The measured cross-sections are shown in Figure 3 where they are compared to NLO pQCD predictions obtained using the CT10 PDF set. The total systematic uncertainty on the cross-section measurement is dominated by the jet energy scale uncertainty. In the lowest $y^{*}$ bin, it is around $10 \%$ at low $m_{12}$ and goes up to $25 \%$ in the highest $m_{12}$ bin. The total systematic uncertainty also increases with $y^{*}$.

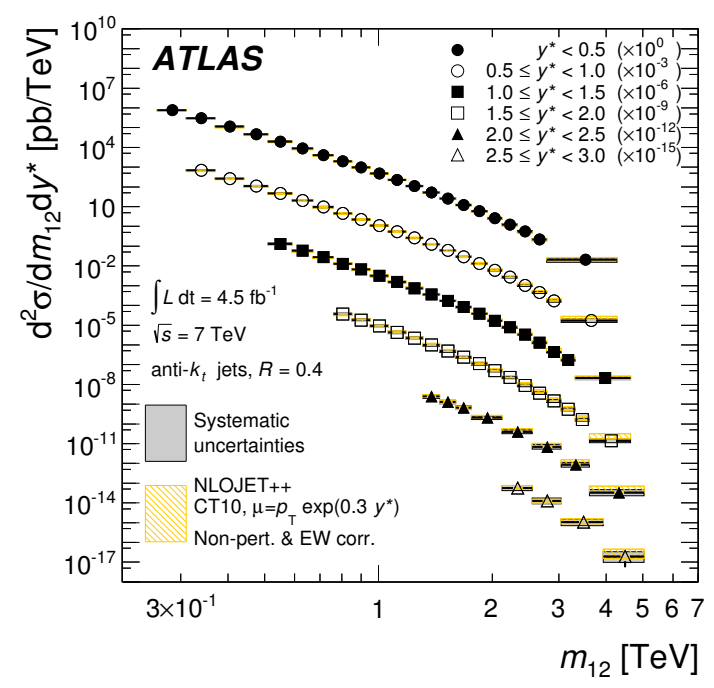

(a)

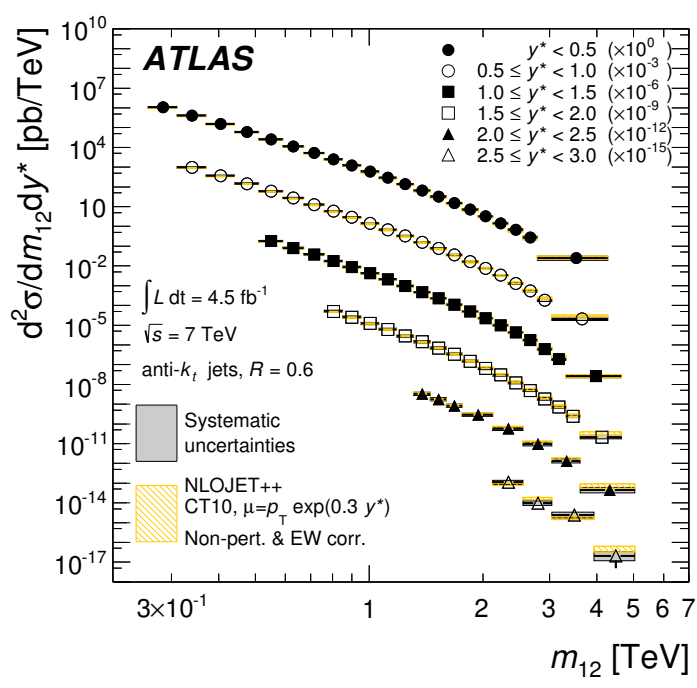

(b)

Figure 3: Double-differential di-jet cross-section as a function of di-jet mass in bins of $y^{*}$ for jets of size parameter (a) $\mathrm{R}=0.4$ and (b) $\mathrm{R}=0.6$. Each $y^{*}$ bin is multiplied by a power of 10 for legibility. The black error bars show the statistical uncertainty (smaller than the data points in most cases) and the grey boxes show the total systematic uncertainty. NLOJET++ pQCD predictions with their uncertainties are shown as yellow hashed boxes [15].

The NLO pQCD predictions describe well the behaviour of the measured cross-sections over many orders of magnitude in the measured cross-section for all $y^{*}$ bins. A subset of the comparisons done with NLO pQCD predictions obtained using other PDFs is shown in Figure 4(a) for the case of jets with $\mathrm{R}=0.6$. Predictions using ABM11 show a strong disagreement with measured cross- 
sections in the three lower $y^{*}$ bins for both jet sizes while those obtained using HERAPDF show small differences in the same range but only for jets with $\mathrm{R}=0.6$.
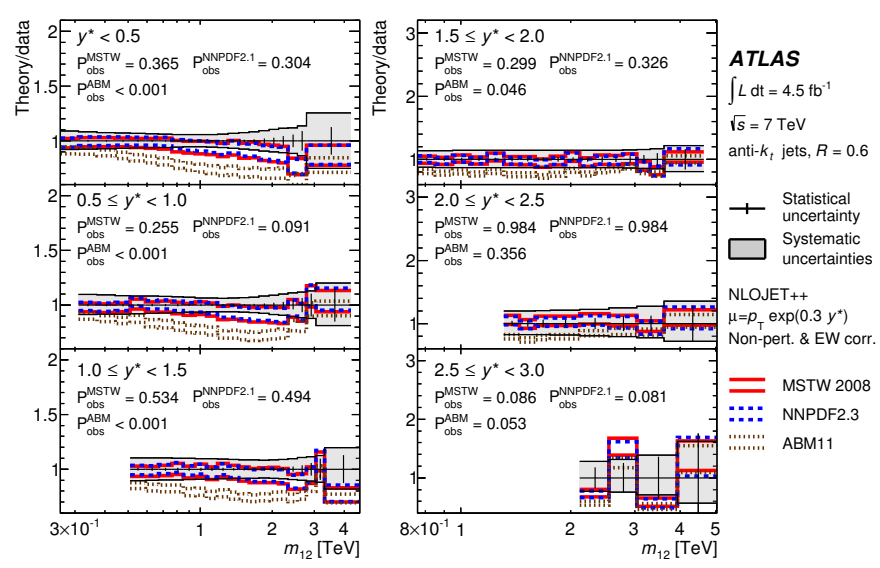

(a)

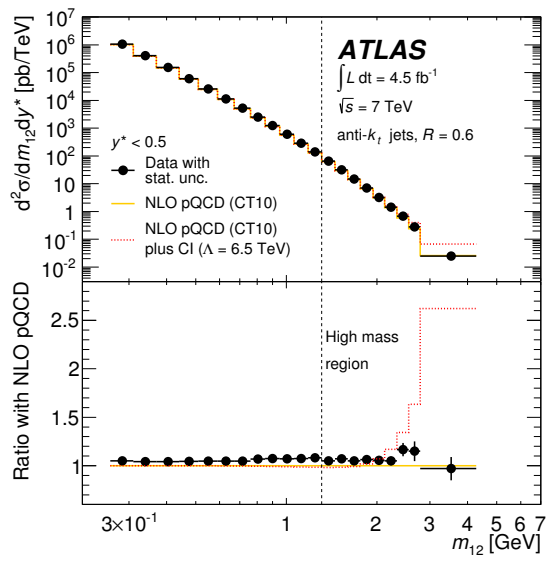

(b)

Figure 4: (a) Ratio of NLO pQCD predictions, obtained with different PDF sets, to the measured doubledifferential di-jet cross-section, shown as a function of $m_{12}$ in six $y^{*}$ bins for jets with size parameters $\mathrm{R}=0.6$. The PDF sets used are MSTW2008 (red), NNPDF2.3 (blue) and ABM11 (brown). The observed p-values are obtained by comparing measurements and predictions over the whole $m_{12}$ range using a generalised $\chi^{2}$ test [16] that allows for inclusion of asymmetric uncertainties [15]. (b) Double-differential di-jet crosssection as a function of di-jet mass in the bin $y^{*}<0.5$ for for jets of size parameter $\mathrm{R}=0.6$. Data is shown with black markers and black error bars indicating the statistical uncertainty on the cross-section measurement. The yellow line shows the Standard Model pQCD NLO prediction using CT10 PDFs while the red dashed line shows the prediction including contact interaction effect with a compositeness scale $\Lambda=6.5 \mathrm{TeV}$ [15].

The effective model of contact interactions as implemented in the CIJET program [17] is used as a benchmark model to illustrate the sensitivity of di-jet double differential cross-section measurements to physics beyond the Standard Model. The main model parameter is the compositeness scale $\Lambda$. This study is performed in the high-mass region $m_{12}>1.31 \mathrm{TeV}$ and in the range $y^{*}<0.5$ which is most sensitive according to theoretical predictions. The effect of the presence of contact interactions at a compositeness scale of $\Lambda=6.5 \mathrm{TeV}$ on the di-jet cross-section in the region of interest is shown in Figure 4(b). Predictions are obtained using a wide range of values of $\Lambda$ and, in the absence of evidence for contact interactions, they are used to set limits on $\Lambda$ using the $C L_{s}$ method [18]. The resulting limits on the compositeness scale $\Lambda$ vary between 6.9 and $7.7 \mathrm{TeV}$ depending on the choice of jet size and PDF.

\section{Tri-jet cross-section}

The double differential tri-jet cross-section [19] is measured as a function of the tri-jet mass $m_{j j j}=\sqrt{\left(p_{1}^{2}+p_{2}^{2}+p_{3}^{2}\right)}$ in bins of the summed rapidity difference of the three leading jets $\left|Y^{*}\right|=$ $\left|y_{1}-y_{2}\right|+\left|y_{2}-y_{3}\right|+\left|y_{1}-y_{3}\right|$. The three leading jets are required to have $p_{\mathrm{T}}>150,100$ and $50 \mathrm{GeV}$, respectively. The measured cross-sections are shown in Figure 5 and compared to NLO pQCD 
predictions obtained with the CT10 PDF set. The systematic uncertainty on the measured crosssections is dominated by the jet energy scale uncertainty and ranges from around $15 \%$ at low $m_{j j j}$ to close to $40 \%$ in the highest $m_{j j j}$ bin.

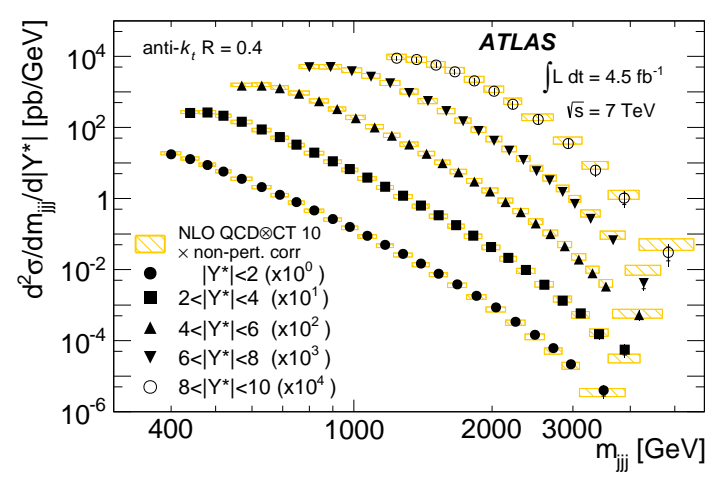

(a)

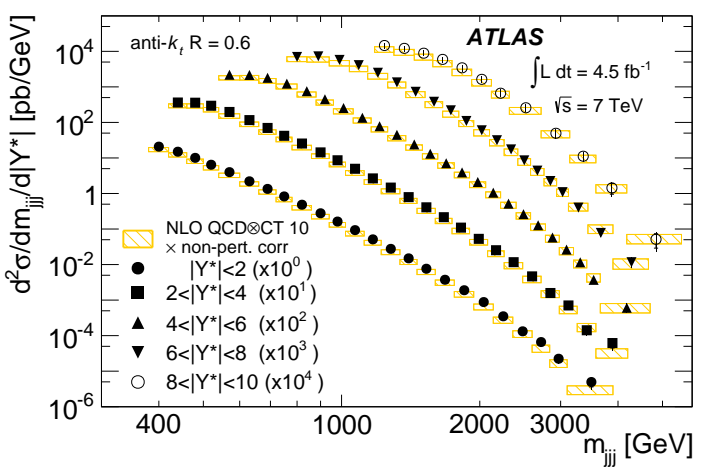

(b)

Figure 5: Double-differential tri-jet cross-section as a function of the tri-jet mass in bins of $\left|Y^{*}\right|$ for jets of size parameter (a) $\mathrm{R}=0.4$ and (b) $\mathrm{R}=0.6$. Each $\left|Y^{*}\right|$ bin is multiplied by a power of 10 for legibility. The error bars show the sum in quadrature of statistical and systematic uncertainties. NLOJET++ pQCD predictions with their uncertainties are shown as yellow hashed boxes [19].

The theoretical predictions describe well the data over seven orders of magnitude in crosssections in all $\left|Y^{*}\right|$ bins. A subset of the comparisons done with NLO pQCD predictions obtained using other PDF sets is shown in Figure 6 for the case of jets with $\mathrm{R}=0.6$. For jets with $\mathrm{R}=0.4$, the data is well matched by predictions obtained from all PDF sets except ABM11. For jets with $\mathrm{R}=0.6$ and all PDFs except ABM11, the predictions are lower than the measurement but within the experimental and theoretical uncertainties. The ABM11 predictions show some disagreements for most of the cross-sections studied and for both jets sizes.

\section{Summary}

The ATLAS experiment has measured the production cross-section for inclusive jets, di-jet and tri-jet events using the full 2011 dataset with a luminosity of $4.5 \mathrm{fb}^{-1}$. This dataset is much larger than the previous 2010 dataset and many improvements have been achieved in the determination and treatment of systematic uncertainties, resulting in a dramatic increase of the measurement precision in all cases. NLO pQCD predictions obtained with NLOJET++ using a variety of PDF sets generally describe well the measured cross-sections. All results contain a full treatment of systematic uncertainties including correlations, so they can be used to constrain the proton PDFs. The di-jet cross-section measurement is sensitive to contact interactions, and limits on the compositeness scale $\Lambda$ are measured to be in the range 6.9-7.7 TeV depending on the choice of jet size and PDF. Further precision measurements of jet production cross-sections using the 2012 ATLAS dataset are ongoing, and will benefit from the larger luminosity of the 2012 dataset as well as the continued effort to reduce systematic uncertainties on the measurements of jet properties. 


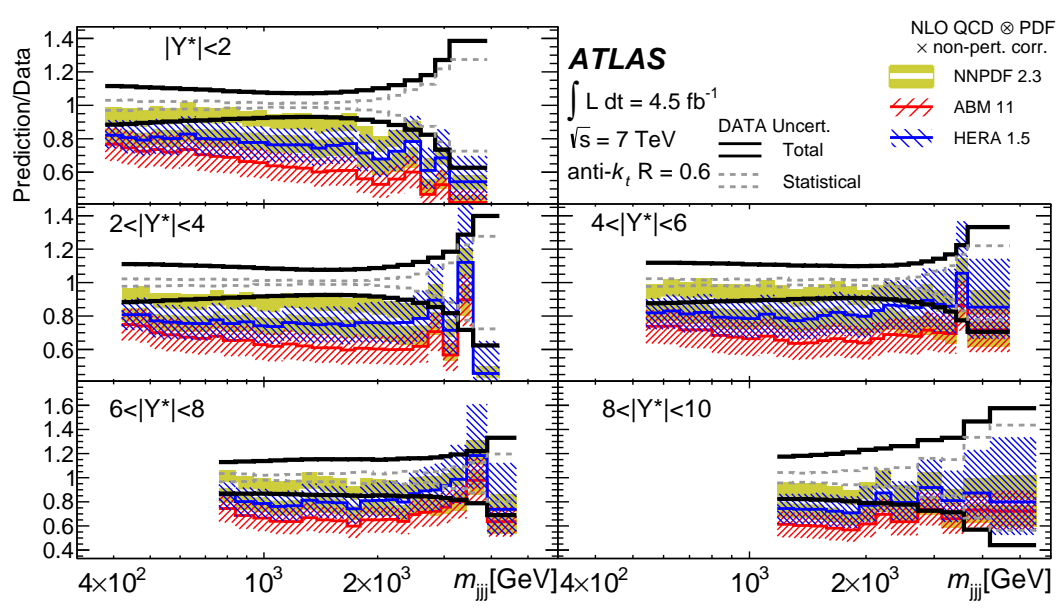

Figure 6: Ratio of NLO pQCD predictions, obtained with different PDF sets, to the measured doubledifferential tri-jet cross-section, shown as a function of $m_{j j j}$ in five $\left|Y^{*}\right|$ bins for jets with size parameters $\mathrm{R}=0.6$. The PDF sets used are NNPDF2.3 (yellow), ABM11 (red) and HERA 1.5 (blue). The dashed grey line is the statistical uncertainty band on the measurement while the black line is the total uncertainty band. The hashed band on the theoretical predictions represents the total theoretical uncertainty [15].

\section{References}

[1] ATLAS Collaboration, JINST 3 (2008) S08003

[2] M. Cacciari, G. Salam, G. Soyez, JHEP 04 (2008) 063

[3] Z. Nagy, Phys. Rev. D 68 (2003) 094002

[4] T. Carli et al., Eur. Phys. J. C 66 (2010) 503

[5] H.-L. Lai et al., Phys. Rev. D 82 (2010) 074024

[6] H1 and ZEUS Collaborations, H1prelim-10-142, ZEUS-prel-10-018

[7] A. D. Martin et al., Eur. Phys. J. C 63 (2009) 189

[8] R. D. Ball et al., Nucl. Phys. B 838 (2010) 136

[9] S. Alekhin, J. Blumlein, S. Moch, Phys. Rev. D 86 (2012) 054009

[10] S. Dittmaier, A. Huss, C. Speckner, JHEP 11 (2012) 095

[11] T. Sjostrand, S. Mrenna, P. Z. Skands, JHEP 05 (2006) 026

[12] M. Bahr et al., Eur. Phys. J. C 58 (2008) 639

[13] B. Malaescu, [arXiv: 0907.3791]

[14] ATLAS Collaboration, JHEP 02 (2015) 153

[15] ATLAS Collaboration, JHEP 05 (2014) 059

[16] G. D’Agostini, Nucl. Instrum. Meth. A 346 (1994) 306; V. Blobel, eConf C 030908 (2003) MOET002

[17] J. Gao, Comput. Phys. Commun. 184 (2013) 2362

[18] A. Read, J. Phys. G 28 (2002) 2693

[19] ATLAS Collaboration, Eur. Phys. J. C 75 (2015) 228 